\title{
June 2014 Pulmonary Case of the Month: "Petrified"
}

\author{
Steven W. Purtle, MD \\ University of Colorado Hospital, Denver, CO \\ steven.purtle@ucdenver.edu
}

\section{History of Present IIIness}

A 52 year old expatriated Iraqi man presents to pulmonary clinic with complaints of chronic dyspnea. While a young man living in Iraq, he had been disqualified from service in the Iraqi Air Force after a screening chest x-ray was found to be abnormal. He had no respiratory symptoms at the time of his disqualification, and he remained asymptomatic for the next twenty five years. Beginning five years ago, he had an insidious onset of breathlessness and exertional intolerance. Over the past several years, he has developed diffuse pleuritic chest pain, non-productive cough, and fatigue. He denies any fevers, chills, night sweats, arthralgias, rash, or visual symptoms. After moving to Denver, Colorado three years ago, he has developed a continuous oxygen requirement of two liters per minute.

\section{$\mathrm{PMH}, \mathrm{FH}, \mathrm{SH}$}

He has no significant past medical or family history. While living in Iraq, he worked as a photographer, but he is currently unemployed. He is a lifelong non-smoker and uses no illicit drugs. He has never had any pets. He denies any exposure to inorganic dusts.

\section{Medications}

None

\section{Physical Examination}

Physical examination reveals a thin, middle-aged man in no acute distress. Vital signs were notable for an oxygen saturation of $90 \%$ on 2 liters per minute of supplemental oxygen. Pulmonary examination was notable for fine inspiratory crackles heard best at the bilateral bases. There was no clubbing or peripheral edema. The remainder of his physical examination was unremarkable.

\section{Laboratory Analysis}

Serum chemistries are within normal limits. Complete blood count shows a normal white blood cell count, hematocrit, and platelet count.

\section{Pulmonary Function Tests}

Pulmonary functions tests are shown in Figure 1. 


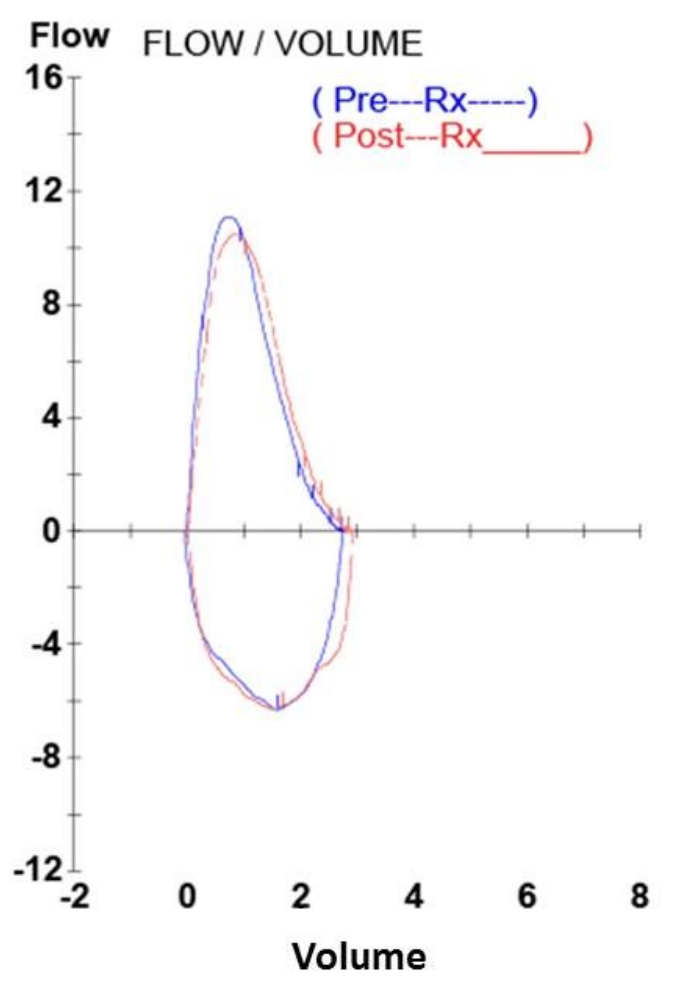

\begin{tabular}{|l|l|}
\hline FEV $_{1} / \mathrm{FVC}$ & 0.92 \\
\hline FEV & $2.55(74 \%)$ \\
\hline FVC & $2.76(64 \%)$ \\
\hline TLC & $4.41(67 \%)$ \\
\hline RV & $1.59(81 \%)$ \\
\hline DLCO & $66 \%$ \\
\hline DLCO/VA & $113 \%$ \\
\hline
\end{tabular}

Figure 1. Admission pulmonary function testing.

\section{Radiography}

A chest X-ray (Figure 2) and chest CT (Figure 3) were performed.

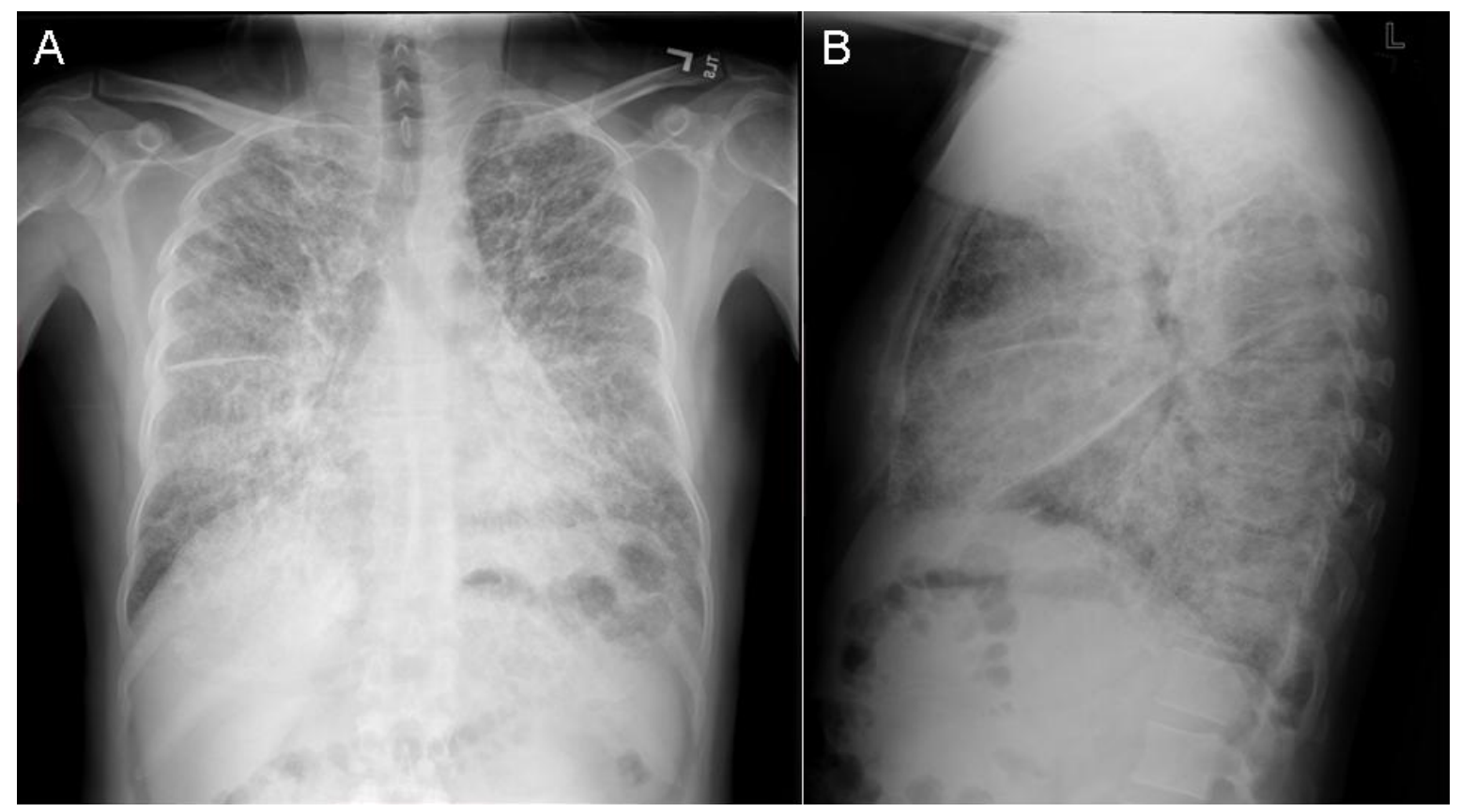

Figure 2. Admission AP (Panel A) and lateral (Panel B) chest X-ray. 

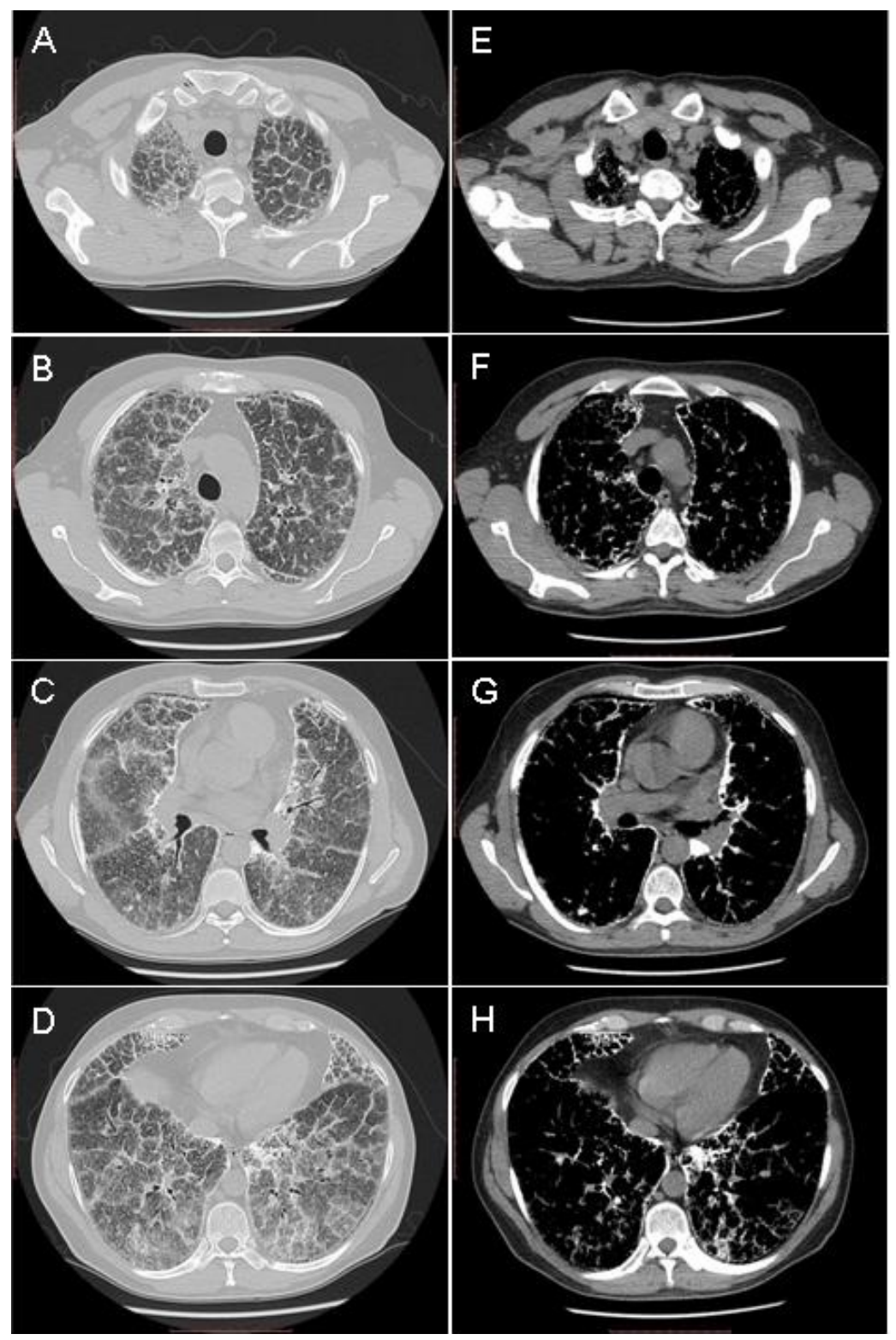

Figure 3. Static thoracic CT images displayed in lung windows (Panels A-D) and soft tissue windows (Panels E-H).

Which of the following features $\underline{\text { best }}$ describes the pattern seen on the patient's chest CT?

1. Diffuse microcalcifications

2. Honeycombing

3. Mosaicism

4. Patchy ground glass opacifications

5. Pleural plaques 


\section{Correct! \\ 1. Diffuse microcalcifications}

This thoracic CT demonstrates pulmonary microcalcifications with a slight lower lobe predominance. This calcification pattern is frequently likened to the appearance of a "sandstorm." The calcifications obscure the border of the heart and other mediastinal structures. Subpleural cyst formation (distinct from honeycombing) gives the appearance of a "black pleural line" and can occasionally be mistaken for a pneumothorax (1).

Which of the following diseases is least likely to be associated with pulmonary calcification?

1. End-stage renal disease

2. Eosinophilic pneumonia

3. Histoplasmosis

4. Pulmonary alveolar microlithiasis

5. Varicella pneumonia 


\section{Correct! \\ 2. Eosinophilic pneumonia}

Pulmonary calcifications can occur in association with a broad spectrum of disease processes. Broadly speaking, pulmonary calcification can be divided into two categories: diffuse small calcifications and large or confluent calcifications. Diffuse small calcifications typically result from infectious processes such as histoplasmosis, tuberculosis, or healed varicella pneumonia. This pattern can also be seen with pneumoconiosis (such as silicosis), non-malignant metastatic calcification (from end stage renal disease or hyperparathyroidism), or genetic disorders such as pulmonary alveolar microlithiasis. Large or confluent nodular calcifications can result from malignant pulmonary metastasis, amyloidosis, or progressive massive fibrosis. Eosinophilic pneumonia is not one of the disorders classically associated with calcification (2).

Which of the following is most likely to be found on surgical lung biopsy?

1. Dendriform pulmonary ossification

2. Hyalinized pulmonary nodules with positively birefringent particles on polarized light microscopy

3. Non-small cell adenocarcinoma

4. Numerous calcified granulomas

5. Small spherical, concentrically layered calcifications within alveoli 


\section{Correct! \\ 5. Small spherical, concentrically layered calcifications within alveoli}

The patient described in this case was found to have pulmonary alveolar microlithiasis based upon his hallmark radiographic findings and surgical lung biopsy.

Pulmonary alveolar microlithiasis is a genetic disorder caused by one of several mutations in the gene that encodes the type IIb sodium phosphate cotransporter protein. This protein serve to transport free phosphate liberated during the breakdown of surfactant from the alveolar space into type II alveolar cells. When this protein is absent or dysfunctional, phosphate accumulates in the alveolar space. When a saturation point is reached with intraalveolar calcium, spherical calcium phosphate crystals ("microliths") form. Mutations in the SCL34A2 are responsible for the disease, which is inherited in an autosomal recessive fashion and found with particular frequency in large consanguineous Middle Eastern families $(3,4)$.

Key radiographic findings, discussed previously, include "sandstorm" pattern of microcalcifications, subpleural cyst formation (producing a "black pleural line"), and obscuration of the heart and mediastinal border by calcifications. Pulmonary physiology shows restrictive spirometry and poor diffusion capacity. There is often a marked discrepancy between the severity of the radiographic findings and clinical symptoms, and many cases are discovered incidentally. Pathology shows pathognomonic microliths, which are characterized by concentrically laminated spheres of calcium phosphate, typically about one micron in size.

There are no proven medical treatments for pulmonary alveolar microlithiasis, although corticosteroids and bisphosphonates have been used in an attempt to arrest further calcification. Successful single and bilateral lung transplantation has been reported and is the only definitive treatment known for pulmonary alveolar microlithiasis (5).

\section{References}

1. Abdalla G, Marchiori E, Zanetti G, et al. Pulmonary alveolar microlithiasis: a case report with emphasis on imaging findings. Case Rep Med. [CrossRef] [PubMed]

2. Marchiori E, Souza AS Jr, Franquet T, Müller NL. Diffuse high-attenuation pulmonary abnormalities: a pattern-oriented diagnostic approach on high-resolution CT. AJR Am J Roentgenol. 2005;184(1):273-82. [CrossRef] [PubMed]

3. Jönsson $A \mathrm{~L}$, Simonsen U, Hilberg O, Bendstrup E. Pulmonary alveolar microlithiasis: two case reports and review of the literature. Eur Respir Rev. 2012;21(125):249-56. [CrossRef] [PubMed]

4. Huqun, Izumi S, Miyazawa $\mathrm{H}$, et al. Mutations in the SLC34A2 gene are associated with pulmonary alveolar microlithiasis. Am J Respir Crit Care Med. 2007;175(3):2638. [CrossRef] [PubMed]

5. Stamatis G, Zerkowski HR, Doetsch N, Greschuchna D, Konietzko N, Reidemeister JC. Sequential bilateral lung transplantation for pulmonary alveolar microlithiasis. Ann Thorac Surg. 1993;56(4):972-5. [CrossRef] [PubMed] 